\title{
Vaginal Pelvic Organ Prolapse Repair Using Mesh: Let's Welcome Science into the Mesh Debate
}

\author{
Frank Van der Aa *, Dirk De Ridder \\ Department of Urology, University Hospitals KU Leuven, Leuven, Belgium
}

In this issue of European Urology, Lucot and colleagues [1] report on PROSPERE, a randomized trial comparing the safety of anterior compartment repair using mesh either via transvaginal mesh repair (TVM) or a laparoscopic abdominal approach (LS). The article offers several insights in this highly debated field.

First, this is indeed one of the first studies that offers level 1 evidence for cystocele repair via an abdominal laparoscopic approach. Although only patients who presented with "primary cystocele" were included in the study, in both cohorts (LS and TVM) approximately 20\% underwent concomitant hysterectomy or apical suspension and 55\% underwent a posterior compartment repair (88\% in the LS group and 23\% in the TVM group). This is striking since it is known that the combination of TVM and vaginal hysterectomy leads to a higher rate of mesh exposure [2]. Moreover, $32 \%$ of the total cohort underwent simultaneous surgery for stress urinary incontinence (SUI). This finding also shows that a large proportion of patients presenting with primary cystocele as their chief complaint will have multicompartment problems, and that "simple cystocele" is rare.

The second learning point is that in experienced hands, the use of mesh has lower complication rates than often reported. After TVM, 2.3\% of patients had vaginal mesh exposure and $0.8 \%$ had bladder erosion at $12 \mathrm{mo}$. No mesh-related problems were found in the LS group at 12 mo. Thus, LS does offer a safer solution than TVM. Conversely, serious complications such as rectal or vascular injury and a need for blood transfusion were rare but slightly higher in the LS group.

This trial does not give us a clear answer on whether the use of TVM is "acceptable". Throughout the world, TVM use is heavily debated. The occurrence of mesh-related complications (not least persisting pain and dyspareunia) and the high reoperation rate are the main arguments against mesh use.

Among the 128 patients who underwent TVM in this prospectively followed cohort, two (1.6\%) experienced persistent de novo pain, while preoperative dyspareunia was ameliorated in one patient. In both groups, an important proportion of patients reported dyspareunia or apareunia due to pain. In the TVM group, this rate increased to $30 \%$. In a trial in a similar patient group (primary cystocele), Vollebregt et al [3] compared native tissue repair versus TVM. They reported a $9 \%$ rate of de novo dyspareunia for native tissue repair versus 15\% in the TVM group. In that specific study, baseline dyspareunia (present in 15/63, 24\%) was more likely to disappear after native tissue repair than after TVM. Only five patients in the TVM group were sexually active before the surgery, which limits the impact of these findings. It seems that every patient undergoing prolapse repair using mesh should be warned about this possible side effect and that TVM should be avoided in sexually active patients because of high dyspareunia rates. Conversely, among sexually inactive women, TVM can be used with acceptable safety margins in the hands of an experienced surgeon.

The reoperation rate at $1 \mathrm{yr}$ was significantly lower in the LS group (4.7\%) than in the TVM group (10.9\%). These reoperations included surgeries for SUI treatment. Half of the reoperations for complications in the TVM cohort were minor procedures at the outpatient clinic under local anesthesia. These figures confirm that TVM use is associated with mesh-specific complications that may require additional procedures under local $(3.9 \%)$ or general $(4.7 \%)$

DOI of original article: https://doi.org/10.1016/j.eururo.2018.01.044.

* Corresponding author. Department of Urology, University Hospitals KU Leuven, Herestraat 49, 3000 Leuven, Belgium. Tel. +32 16 346930; Fax: +32 16346931 .

E-mail address: frank.vanderaa@uzleuven.be (F. Van der Aa). 
anesthesia after 1-yr follow-up. This knowledge should obviously be taken into account when considering the use of mesh via the vaginal route. These numbers will most likely increase after longer follow-up.

The results of this trial are not necessarily applicable in real life setting. This is a well-designed randomized trial that, as it should, clearly delineates a very specific patient population. Although this adds to the strengths of the study from a scientific perspective, the conclusions of the paper should only be translated into clinical practice with caution. Among the 925 patients assessed for the study, 663 were excluded for various reasons. The results presented are only applicable to a subset of the population a physician sees at the outpatient clinic.

We should not make the same mistake again: prolapse repair is not easy surgery! Expertise is mandatory for diagnosis and surgical indication and for the surgery itself (whether vaginally or abdominally, using native tissue or mesh). For diagnosis and indication, a multidisciplinary approach is desirable [4]. For the surgery, the authors carefully selected highly experienced surgeons in highvolume centers in France. This is reflected in the generally low complication rate at $1 \mathrm{yr}$. We know that the learning curve for laparoscopic sacrocolpopexy is approximately 60 surgeries [5]. Even if it can be brought down via laboratory training, this number of surgeries is quite high for a relatively infrequent operation [6].

Centers that offer prolapse repair should offer multidisciplinary advice and a broad armamentarium for pelvic organ prolapse (POP) treatment. Personalized or categorized medicine should be offered in surgery as in medical treatment. A patient with POP could be offered vaginal native tissue, vaginal mesh, or abdominal mesh repair, depending on factors such as whether the POP is primary or recurrent; the risk of recurrence; contraindication for mesh use; history and comorbidities; desire for future sexual activity; and the need for additional surgeries (other compartments or SUI).
As shown in the PROSPERE trial, in a subset of these patients the decision might change during the treatment phase, as $5.4 \%$ of patients received TVM because LS was not feasible or safe in expert hands.

The PROSPERE trial is a very welcome study in a field in which scientific data are sparse and medicolegal arguments have overtaken the debate. Although this study does not solve all aspects of the debate, it certainly adds value.

Conflicts of interest: Frank Van der Aa is proctor and consultant for Coloplast and has lectured for Boston Scientific. Dirk De Ridder is a consultant for Coloplast and Boston Scientific.

\section{References}

[1] Lucot JP, Cosson M, Bader G, et al. Safety of vaginal mesh surgery versus laparoscopic mesh sacropexy for cystocele repair: results of the Prosthetic Pelvic Floor Repair randomized controlled trial. Eur Urol 2018;74:167-76. http://dx.doi.org/10.1016/j.eururo.2018.01.044.

[2] Barski D, Otto T, Gerullis H. Systematic review and classification of complications after anterior, posterior, apical, and total vaginal mesh implantation for prolapse repair. Surg Technol Int 2014;24:217-24.

[3] Vollebregt A, Fischer K, Gietelink D, Van Der Vaart CH. Primary surgical repair of anterior vaginal prolapse: a randomised trial comparing anatomical and functional outcome between anterior colporrhaphy and trocar-guided transobturator anterior mesh. Br J Obstet Gynaecol 2011;118:1518-27. http://dx.doi.org/10.1111/j. 1471-0528.2011.03082.x.

[4] Chapple CR, Cruz F, Deffieux X, et al. Consensus statement of the European Urology Association and the European Urogynaecological Association on the use of implanted materials for treating pelvic organ prolapse and stress urinary incontinence. Eur Urol 2017;72:424-32. http://dx.doi.org/10.1016/j.eururo.2017.03.048.

[5] Claerhout F, Roovers JP, Lewi P, Verguts J, De Ridder D, Deprest J. Implementation of laparoscopic sacrocolpopexy-a single centre's experience. Int Urogynecol J 2009;20:1119-25. http://dx.doi.org/10. 1007/s00192-009-0914-X.

[6] Deprest J, Krofta L, Van Der Aa F, et al. The challenge of implementing laparoscopic sacrocolpopexy. Int Urogynecol J Pelvic Floor Dysfunct 2014;25:1153-60. http://dx.doi.org/10.1007/s00192-014-2398-6. 